\title{
Surgery combined with antibiotics for the treatment of endogenous endophthalmitis caused by liver abscess
}

\author{
Yue Wang, Xue Wang and Yu Di
}

\begin{abstract}
Backgrounds: Endogenous endophthalmitis is a serious disease caused by intraocular infection that can rapidly progress to cause blindness. This study evaluated the clinical features, surgical and antibiotics treatment strategies, and treatment outcomes in patients with endophthalmitis caused by liver abscess.

Methods: Between April 2014 and April 2019, the clinical data of 16 patients (19 eyes) with endophthalmitis associated with liver abscess who underwent surgery at Shengjing Hospital were retrospectively analyzed. Furthermore, we evaluated the final visual outcomes in the patients to determine the efficacy of surgery.

Results: Fifteen patients (18 eyes) underwent intravitreal injection followed by vitrectomy after admission. One patient ( 1 eye) only underwent intravitreal injection. Of the 16 patients, 3 patients ( 3 eyes) had recurrent intraocular inflammation and eventually underwent evisceration. Systemic antibiotics were administered for all patients based on the results of vitreous humor culture, blood culture, and antibiotic susceptibility tests. Outpatient follow-ups were performed until the patients were stable ( 6 months). Of the 19 eyes, 1 eye (5\%) had visual acuity restored to 20/200, 6 eyes (31\%) had visual acuity restored to counting fingers (CF), 2 eyes (11\%) had visual acuity restored to hand motion (HM), 4 eyes (22\%) showed only light perception (LP), and the remaining 6 eyes (31\%) showed no light perception (NLP). Drug susceptibility tests suggested that the carbapenems exhibited significant effects in the inflammatory reaction.

Conclusion: Endogenous endophthalmitis caused by liver abscess is a very serious condition, and the final visual outcome is poor. Timely surgical intervention combined with antibiotic treatment is essential, and the primary disease must be treated to control disease progression at the earliest.
\end{abstract}

Keywords: Endogenous endophthalmitis, Klebsiella pneumoniae, Liver abscess, Vitrectomy, Visual acuity

\section{Background}

Endophthalmitis is an ocular disease that can lead to serious visual acuity damage $[1,2]$. Endogenous endophthalmitis as a result of liver abscess is caused by the hematogenous spread of pathogenic bacteria from the liver abscess, which passes through the blood-retinal

\footnotetext{
*Correspondence: diyujsdxt@163.com

Department of Ophthalmology, Shengjing Hospital of China Medical University, Heping district, Sanhao Road 36, Shenyang 110004, People's Republic of China
}

barrier, leading to infection of the eye [3]. In recent years, many cases have been reported in Asia, with Klebsiella pneumoniae being the most common causative agent $[4,5]$. Since the disease is relatively occult during the early stage, it can rapidly infect intraocular tissues, resulting in irreversible damage to the photoreceptor cells in the retina. This causes irreversible damage to the eye, leading to a significant impact on visual function, and can also potentially be life-threatening [6-8]. Therefore, prompt management during the early stage is

(c) The Author(s). 2020 Open Access This article is licensed under a Creative Commons Attribution 4.0 International License, which permits use, sharing, adaptation, distribution and reproduction in any medium or format, as long as you give appropriate credit to the original author(s) and the source, provide a link to the Creative Commons licence, and indicate if changes were made. The images or other third party material in this article are included in the article's Creative Commons licence, unless indicated otherwise in a credit line to the material. If material is not included in the article's Creative Commons licence and your intended use is not permitted by statutory regulation or exceeds the permitted use, you will need to obtain permission directly from the copyright holder. To view a copy of this licence, visit http://creativecommons.org/licenses/by/4.0/ The Creative Commons Public Domain Dedication waiver (http://creativecommons.org/publicdomain/zero/1.0/) applies to the data made available in this article, unless otherwise stated in a credit line to the data. 
essential to prevent permanent blindness and mortality. In this study, we evaluated the clinical features, surgical strategies, pathogenic bacterial features, and visual outcomes in patients with endophthalmitis caused by liver abscess.

\section{Methods}

\section{Study design and clinical data collection}

We retrospectively analyzed patients with liver abscessassociated endophthalmitis who were admitted to the Department of Ophthalmology of Shengjing Hospital of China Medical University between April 2014 and April 2019. Patients with the following characteristics were included: diagnosis of endophthalmitis by operation and examination and liver abscess confirmed by imaging. Patients with the following risk factors were excluded: history of keratitis, history of intraocular surgery, trauma, or glaucoma [9]. Finally, 16 patients (19 eyes) of 72 patients with endogenous endophthalmitis were included in the study. Clinical data collected included patient demographic information, length of hospitalization, department admitted after initial diagnosis, medical history, patient symptoms, treatment strategy, and degree of visual acuity correction after surgery. All procedures involving human participants were conducted in accordance with the tenets of the Declaration of Helsinki. The study design was approved by the Ethics Committee of the Department of Ophthalmology of Shengjing Hospital.

\section{Treatment strategy}

Once a diagnosis of endogenous endophthalmitis was confirmed by ocular ultrasonography and fundus examination in patients who visited the ophthalmology clinic or after consultation with the Department of Hepatobiliary Surgery, the patients were treated through surgery. Patients either underwent vitrectomy using silicone oil combined with an intravitreal injection of antibiotics, or received an intravitreal injection alone. Of the 19 eyes, Only 1 patient (1 eye) was intravitreal injection alone. Depending on the treatment strategy and the surgeon's level of experience, patients received an intravitreal injection of $1 \mathrm{mg} / 0.1 \mathrm{ml}$ vancomycin, $2.25 \mathrm{mg} / 0.1 \mathrm{ml}$ ceftazidime, and $0.2 \mathrm{mg} / 0.1 \mathrm{ml}$ dexamethasone. Tobramycin and dexamethasone were administered as topical eye drops. The administration of systemic antibiotics was adjusted based on the results of the antibiotic susceptibility tests. Outpatient follow-ups were performed for 6 months, until the patients were stable.

\section{Statistical analysis}

The visual acuity was recorded using logMAR as follows: counting fingers $(\mathrm{CF})=1.7$ logMAR, hand motion $(\mathrm{HM})=2.0 \operatorname{logMAR}$, light perception $(\mathrm{LP})=2.3 \log \mathrm{MAR}$, no light perception $(\mathrm{NLP})=3.0 \log$ MAR $[10,11]$. The visual acuity of patients who underwent evisceration was considered NLP. All data were analyzed using SPSS17.0. Statistical differences between preoperative and postoperative visual acuities were analyzed using Single sample Wilcoxon test, with a significance level set at $P<0.05$.

\section{Results}

\section{Baseline patient characteristics}

Of the 16 patients enrolled in this study, the mean age was 56 years ( $40-67$ years). There were 9 (56\%) male patients and 7 (44\%) female patients. Among the 19 eyes investigated, 10 eyes (53\%) were right eyes, and 9 eyes (47\%) were left eyes. The mean length of hospital stay was 18.75 days (6-37 days). Nine patients (56\%) had an initial diagnosis of endophthalmitis. Six patients (38\%) had liver abscess and 1 patient (6\%) had fever as initial symptom. Significant systemic medical problems included hypertension in 6 patients (38\%) and diabetes mellitus in 9 patients (56\%). The characteristics are listed in Table 1.

\section{Liver abscess clinical characteristics}

Patients with a liver abscess displayed notable fever and upper right abdominal pain. An emergency blood test revealed a significant elevation of white blood cells and neutrophils, and $\mathrm{C}$-reactive protein levels tested at the emergency department were also significantly elevated. Abdominal CT showed low-density lesions at various locations in the liver, which were suggestive of an intrahepatic abscess (Fig. 1a). The diameter of the liver abscess was $2.1-8.8 \mathrm{~cm}$, whereas the average diameter was $(4.17 \pm 2.06) \mathrm{cm}$.

Table 1 Characteristics of the patients

\begin{tabular}{ll}
\hline Characteristics & Values \\
\hline Age (years) & $56(40-67)$ \\
Gender & \\
$\quad$ Male $(n, \%)$ & $9(56 \%)$ \\
$\quad$ Female (n,\%) & $7(44 \%)$ \\
Left/right side & \\
$\quad$ Right (n,\%) & $10(53 \%)$ \\
Left (n,\%) & $9(47 \%)$ \\
Length of hospital stay (days) & $18(6$ to 37$)$ \\
Initial diagnosis & \\
$\quad$ Endophthalmitis (n,\%) & $9(56 \%)$ \\
Liver abscess (n,\%) & $6(38 \%)$ \\
Fever (n,\%) & $1(6 \%)$ \\
Hypertension & $6(38 \%)$ \\
Diabetes & $9(56 \%)$ \\
\hline
\end{tabular}



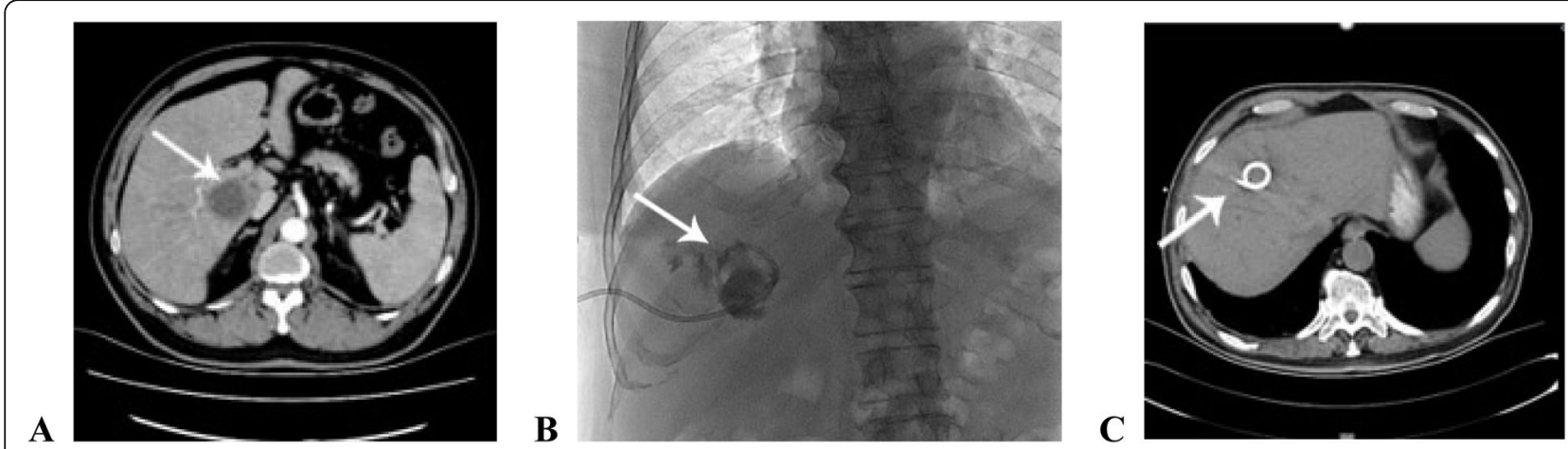

Fig. 1 Clinical features of liver abscess. a Contrast-enhanced abdominal CT image of liver abscess suggestive of intrahepatic abscess. b Plain abdominal radiograph of the liver abscess after drainage, showing that the drainage catheter was well-positioned, and the abscess had subsided. c Contrast-enhanced abdominal CT image of the liver abscess after drainage, showing that the drainage catheter was well-positioned, and the abscess had subsided

All patients underwent percutaneous liver abscess drainage, and received a follow-up plain abdominal radiography (Fig. 1b) and contrast-enhanced abdominal CT (Fig. 1c), both of which showed that the drainage catheter was well-positioned and the abscess had subsided.

\section{Clinical features of endophthalmitis}

Slit-lamp bio-microscopic examination revealed conjunctival hyperemia, corneal edema, and inflammation of the anterior chamber, accompanied by discernable pus accumulation or fibrinous exudates (Fig. $2 \mathrm{a}$ and b). Funduscopic examination did not form a sharp image of the retina. Three-dimensional ultrasound imaging of the eye showed turbidity in the form of streaks, dots, or floccules in the vitreous humor, which were suggestive of endophthalmitis (Fig. 2c). During the vitrectomy, pus accumulation in the anterior chamber and vitreous humor was observed, and a large amount of purulent exudate
A

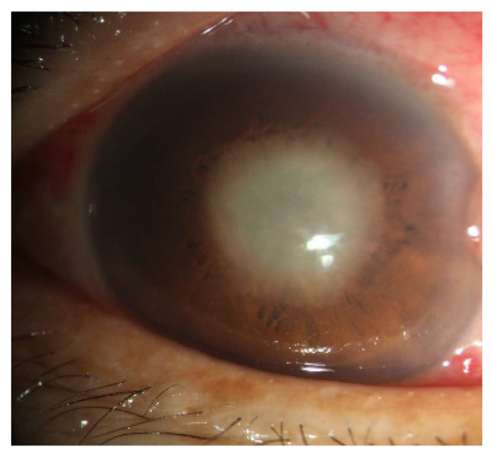

B

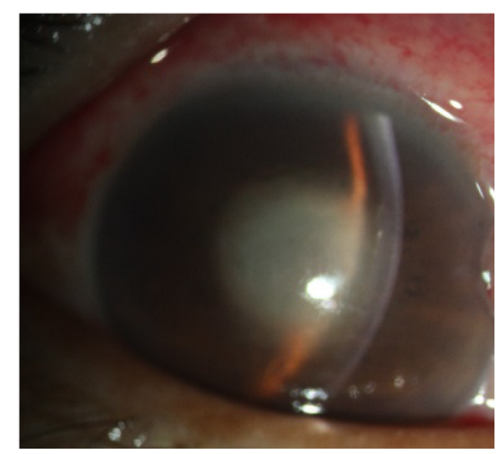

C

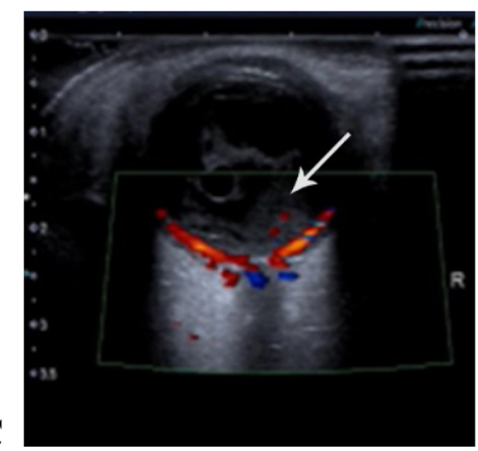

D

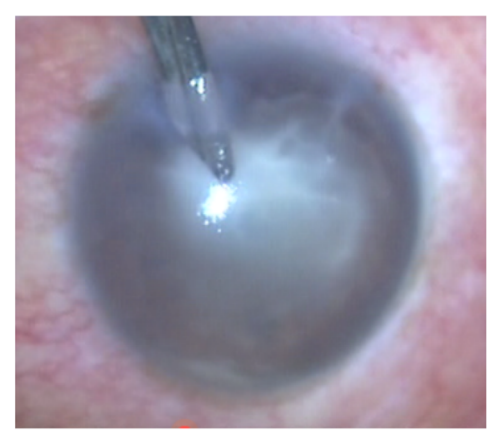

E

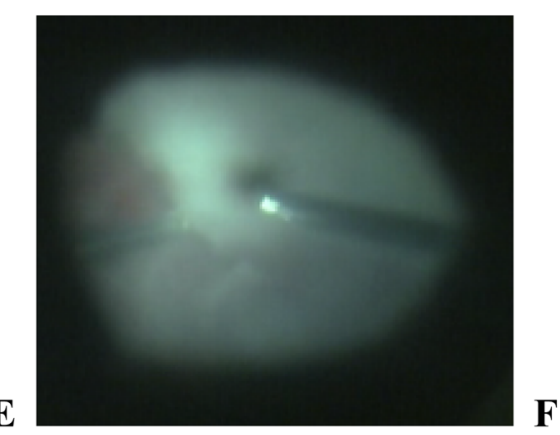

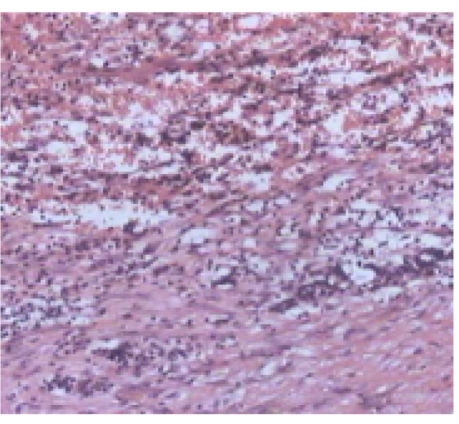

Fig. 2 Clinical features of liver endophthalmitis. a and b Biomicroscopic examination revealing conjunctival hyperemia, corneal edema, and inflammation of the anterior chamber, accompanied by discernable pus accumulation or fibrinous exudates. c Three-dimensional ultrasound imaging of the eye showing turbidity in the form of streaks, dots, or floccules in the vitreous humor. d-e Purulent exudate was observed in the anterior chamber and vitreous humor. Tight adhesion of fibrinous exudate was observed in the vitreous cavity. $\mathbf{f}$ Pathological image of eye biopsy showing extensive neutrophil infiltration and piecemeal necrosis 
was present in the vitreous cavity (Fig. 2d-e). During vitrectomy, it was observed that the retina was light in color, and the fibrinous exudate was tightly adherent. An eye biopsy for patients who underwent evisceration showed that the eye was partially lined with squamous epithelium, with infiltration of neutrophils cells underneath. The lining contralateral to the squamous epithelium showed extensive neutrophil infiltration and piecemeal necrosis (Fig. 2f).

\section{Microbial culture}

Microbial cultures were performed on the drainage fluid, blood, and vitreous humor of all patients. Klebsiella pneumoniae was identified in $8(50 \%)$ patients, Serratia marcescens in 1 (6\%) patient, Bacillus licheniformis in 1 (6\%) patient, and Staphylococcus warneri in 1 (6\%) patient. For the remaining 5 (32\%) patients, no apparent bacteria or fungus could be identified in culture. Results of the postoperative blood and drug susceptibility tests suggested that the carbapenems, such as Imipenem and Meropenem, exhibited significant effects in the inflammatory reaction. The results are shown in Table 2.

\section{Restoration of visual activity}

Sixteen eyes received ophthalmologic surgery for treatment for primary disease, combing with postoperative intravenous antibiotics, which effectively controlled inflammation. Three eyes had recurrent inflammation, and evisceration was subsequently performed. One eye $(5 \%)$ had visual acuity restored to 20/200, 6 eyes (31\%) had visual acuity restored to CF, 2 eyes (11\%) had visual acuity restored to HM, 4 eyes (22\%) showed only LP, and the remaining 6 eyes (31\%) eventually showed NLP. Single sample Wilcoxon test to compare the preoperative and postoperative visual acuities to determine treatment efficacy showed a statistically significant difference $(z=$ $-2.598, P<0.05)$. The results are shown in Table 3.

\section{Discussion}

Recent studies have shown that endophthalmitis caused by liver abscess accounts for an increasing percentage of endogenous endophthalmitis cases each year, particularly in East Asia [12]. A study on endogenous endophthalmitis conducted in Korea showed that 25\% of primary lesions were liver abscesses [13], whereas a study in Taiwan found that $53 \%$ of primary lesions were liver abscesses, and $61 \%$ of cases were caused by Klebsiella pneumoniae, and showed the trend of drug resistance [14]. In the present study, microbial cultures of the vitreous humor, as well as blood cultures were obtained from all patients. Bacteria could be identified in 11 (69\%) cases, 8 (50\%) of which were positive for Klebsiella pneumoniae. In Asia, the incidence rate of multidrugresistant and hypervirulent Klebsiella pneumoniae strains increased [15]. We found that there were 3 patients with carbapenem resistance; however, the patients were not included in our study because of the surgery and the discontinued visiting of the patients.

One patient's endophthalmitis was caused by Serratia marcescens, and the final visual outcome of the 2 eyes was NLP. Endophthalmitis caused by Serratia marcescens is very rare, where most of the cases progress to NLP. Most of the reported cases have a history of interventional surgery, oral surgery, and intravenous drug use [16]. Both liver abscess and endophthalmitis caused by Staphylococcus walleriae and Bacillus licheniformis are uncommon, implying they should be paid more attention in the clinics. The ideal condition for performing a bacterial culture is during the absence of antibiotic treatment following disease onset. However, due to the rapid

Table 2 Culture and drug sensitivity tests of endogenous endophthalmitis patients

\begin{tabular}{llll}
\hline Patient & Pathogenic bacteria & \multicolumn{1}{c}{ Culture } & Antibiotic resistance \\
\hline 1 & V/ B/ D & AMP, CFZ, TE, SSS \\
2 & Klebsiella pneumoniae & $+/-/ \mathrm{NA}$ & AMP, AZT, CIP, CRO, FEP, CFZ, NIT, LVX \\
3 & Klebsiella pneumoniae & $+/+/ \mathrm{NA}$ & AMP \\
4 & Klebsiella pneumoniae & $+/-/ \mathrm{NA}$ & $\mathrm{CTT}, \mathrm{NIT}, \mathrm{CFZ}$, \\
5 & Klebsiella pneumoniae & $+/-/ \mathrm{NA}$ & $\mathrm{AMP}$ \\
6 & Klebsiella pneumoniae & $+/-/ \mathrm{NA}$ & $\mathrm{AMP}, \mathrm{TE}$ \\
7 & Klebsiella pneumoniae & $+/-/ \mathrm{NA}$ & $\mathrm{AMP}$ \\
8 & Klebsiella pneumoniae & $+/-/+$ & $\mathrm{NA}$ \\
9 & Klebsiella pneumoniae & $\mathrm{NA} /-/+$ & $\mathrm{CTT}, \mathrm{NIT}, \mathrm{CFZ}, \mathrm{SSS}$ \\
10 & Serratia marcescens & $+/+/+$ & $\mathrm{NA}$ \\
11 & Bacillus licheniformis & $\mathrm{NA} /-/+$ & $\mathrm{NA}$ \\
\hline
\end{tabular}

$V$ Vitreous; B Blood; D drainage fluid; NA Not available; AMP Ampicillin; CFZ Cefazolin; TE Tetracycline; SSS Sulfonamides; AZT Aztreonam; CIP Ciprofloxacin; NIT Nitrofurantoin; CTT Cefotetan; FEP Cefepime; CRO Ceftriaxone; LVX Levofloxacin 
Table $\mathbf{3}$ Visual acuity of the endogenous endophthalmitis patients

\begin{tabular}{lll}
\hline Visual acuity & $\begin{array}{l}\text { Initial visual } \\
\text { activity }\end{array}$ & $\begin{array}{l}\text { Final visual } \\
\text { outcome }\end{array}$ \\
\hline Better than 20/200 & $1(5 \%)$ & $1(5 \%)$ \\
Counting fingers (CF) & $0(0)$ & $6(31 \%)$ \\
Hand motion (HM) & $3(16 \%)$ & $2(11 \%)$ \\
Light perception (+) (LP) & $10(53 \%)$ & $4(22 \%)$ \\
Light perception (-) (NLP) & $5(26 \%)$ & $6(31 \%)$ \\
LogMAR Median (IQR) & $2.3(2.3-3.0)$ & $2.3(1.7-3.0)$ \\
\hline
\end{tabular}

progression of the disease, and the lack of a standardized antibiotic treatment strategy for endogenous endophthalmitis, it is difficult to control this condition, and the results of the culture may be unreliable [17]. Therefore, for effective management of endogenous endophthalmitis, blood samples should be collected before starting antibiotic treatment [18].

Liver abscess-associated endophthalmitis can significantly impact the visual acuity of patients, and has a poor prognosis. A study conducted in Southern California investigating endophthalmitis caused by Klebsiella pneumoniae showed that approximately half of the patients required enucleation [19]. It has been reported that early use of antibiotics combined with timely vitrectomy in patients with endophthalmitis can effectively improve the visual acuity [20]. In the present study, we found that while the condition of the patients with liver abscess-associated endophthalmitis could be improved by vitrectomy combined with intravitreal injection, the overall prognosis was poor. Endogenous endophthalmitis caused by liver abscess is a metastatic infection, in which inflammation occurs rapidly and pathogenic bacteria can invade the inner eyes in a short period of time, which makes surgery more difficult [2]. In this study, 56\% (9 patients) with endogenous endophthalmitis were initially diagnosed with endophthalmitis, other $44 \%$ (7 patients) were only a liver abscess and fever. Eye symptoms are easily neglected when rescuing critical symptoms and focusing on systemic conditions, thereby missing the opportunity for early diagnosis and effective treatment. Furthermore, the clinical manifestations of liver abscess-associated endophthalmitis are nonspecific, and the condition can easily be misdiagnosed as other diseases, such as uveitis, leading to a delay in diagnosis and treatment. Four patients had NLP vision when they were transferred to an ophthalmology department, and their vision could not be preserved. Therefore, when treating patients with severe liver abscess, especially those in coma, doctors should pay attention to the patients' eyes.

Vitrectomy is effective for the treatment of retinal detachment, vitreous hemorrhage, and diabetic retinopathy. It is also the most commonly used surgical method that has demonstrated a definitive efficacy for the treatment of endophthalmitis [21-23]. Vitrectomy can remove intravitreal inflammatory lesions, bacteria and toxins, and reduce the damage caused by the effect of toxic substances on the retinal function [24]. It may also rescue vitreous transparency and reduce or avoid tractional retinal detachment $[25,26]$. Intravitreal injection enables the rapid and effective delivery of antibiotics, to achieve high local drug concentrations. This allows the antibiotics to target the pathogenic bacteria more effectively, thereby inhibiting bacterial growth and controlling inflammation. Concurrent intravitreal vancomycin, ceftazidime and dexamethasone are recommended for patients with infectious endophthalmitis caused by pyogenic liver abscess $[27,28]$.According to experimental and clinical observations, most clinicians believe that injection of vancomycin, ceftazidime and dexamethasone into the vitreous cavity is safe and doses not lead to retinal toxicity. Studies have shown that intravitreal antibiotic injection for susceptible pathogens within $48 \mathrm{~h}$ may help in retaining the visual acuity in some patients with liver abscess-associated endophthalmitis, and patients who received antibiotic intervention $48 \mathrm{~h}$ following the disease onset ultimately had poorer visual acuity [29]. Despite prompt treatment, the visual sequelae of endogenous endophthalmitis caused by liver abscess are frequent and outcomes are poor. In the current series of studies, only 6 patients (31\%) achieved CF or better visual acuity at 6 months of follow-up. In addition, because some patients who underwent vitrectomy, are accompanied with bacteremia, postoperative infection is still a high-risk factor for blindness. Three patients in this study underwent enucleation as a result of the infection not effectively controlled in time. Repeated intravitreal and periocular injections of antibiotics and dexamethasone could prevent enucleation [30].Therefore, it is necessary to inject antibiotics intravenously according to the results of drug sensitivity after vitrectomy.

The present study showed that upon diagnosis of endophthalmitis caused by liver abscess, treatment should be initiated immediately and include intravitreal antibiotics injections, and vitrectomy. We concluded that vitrectomy combined with intravitreal injection is effective in the treatment of endogenous endophthalmitis. Further, the active treatment of the primary lesions was critical. The administration of systemic antibiotics should be accompanied by active treatment of surgical abscess drainage. Antibiotics should be selected according to the results of drug sensitivity. Cephalosporins or carbapenems can be selected according to experience before obtaining drug sensitivity results [30, 31].Therefore, The disease was effectively mitigated, and progression to panophthalmitis was controlled. 
Our study had some limitations. Firstly, the limited case data because of the retrospective nature of the study, secondly, the sample size of this study was small. Future studies may include a larger sample size and a multicentered approach.

\section{Conclusion}

Endogenous endophthalmitis caused by liver abscess is a serious disease with rapid progression and poor prognosis. In this study, early intravitreal injection and vitrectomy are important for improving visual acuity. Further, the systematic and thorough treatment of liver abscess is of great significance in preventing the recurrence of endophthalmitis because of liver abscess. Additionally, we suggest that surgeons should pay attention to the symptoms of the eyes while diagnosing and treating patients with liver abscess.

\section{Abbreviations}

CF: Counting fingers; HM: Hand motion; LP: Light perception; NLP: No light perception

\section{Acknowledgements}

Not applicable.

\section{Authors' contributions}

YD designed the study, treated the patient and was responsible for the collection of case data. YW was responsible for writing the manuscript and the data analysis. XW was responsible for the compilation of case data and pictures. All the authors approved the final version of the manuscript.

\section{Funding}

The work was funded by the National Natural Science Foundation of China (81600747), Startup Foundation for Doctors of Liaoning Province (No.201501020).

\section{Availability of data and materials}

All data generated or analyzed during this study are included in this published article (and its supplementary information files).

\section{Ethics approval and consent to participate}

The study design was approved by the Ethics Committee of the Department of Ophthalmology of Shengjing Hospital.

Informed consent was not needed as this was a retrospective study and the information of patients were anonymously reviewed.

\section{Consent for publication}

Not applicable.

\section{Competing interests}

The authors report no conflicts of interest in this work.

Received: 13 April 2020 Accepted: 2 September 2020

Published online: 07 September 2020

\section{References}

1. Shon AS, Bajwa RPS, Russo TA. Hypervirulent (hypermucoviscous) Klebsiella pneumoniae: a new and dangerous breed. Virulence. 2013;4(2):107-18. https://doi.org/10.4161/viru.22718.

2. Tan ST, Su YJ. Pseudopneumoperitoneum caused by klebsiela pneumoniae pyogenic liver abscess. J Emerg Med. 2019;56(6):127-8. https://doi.org/10. 1016/j.jemermed.2019.02.003.

3. Schiedler V, Scott IU, Flynn HW Jr, Davis UL, Benz MS, Miller D. Cultureproven endogenous endophthalmitis: clinical features and visual acuity outcomes. Am J Ophthalmol. 2004;137(4):725-31. https://doi.org/10.1016/j. ajo.2003.11.013..
4. Fujita M, Takahashi A, HiromichiN I, Hayashi M, Okai K, Abe K, et al. Endogenous Endophthalmitis associated with pyogenic liver abscess caused by Klebsiella pneumoniae. Intern Med. 2019;58:2507-14. https://doi.org/10. 2169/internalmedicine.2684-19.

5. Zhou Y, Wang X, Shen J, Lu ZZ, Liu Y. Endogenous Endophthalmitis caused by Carbapenem-resistant Hypervirulent Klebsiella Pneumoniae: a case report and literature review. Ocul Immunol Inflamm. 2019;27(07):1099-104. https:// doi.org/10.1080/09273948.2018.1502786.

6. Shirakawa C, Yanagihara C, Takano S. I shio Y, Mikawa a. endogenous endophthalmitis following staphylococcus aureus meningitis. Clin Neurol. 2019;59(4):185-9. https://doi.org/10.5692/clinicalneurol.cn-001233.

7. Ho V, Ho LY, Ranchod TM, Drenser KA, Williams GA, Garretson BR. Endogenous methicillin-resistance staphylococcus aureus endophthalmitis. Retina. 2011;31(3):596-601. https://doi.org/10.1097/IAE.0b013e3181ecccfo.

8. Kansal V, Rahimy E, Garg S, Dollin M. Endogenous methicillin-resistant Staphylococcus aureus endophthalmitis secondary to axillary phlegmon: a case report. Can J Ophthalmol. 2017;52(3):97-9. https://doi.org/10.1016/j.jcjo. 2016.11.016.

9. Gounder PA, Hille DM, Khoo YJ, Phagura RS, Chen FK. Endophthalmitis in Western Australia :a sixteen-year retrospective study. Retina. 2020;40:908-18. https://doi.org/10.1097//AE.0000000000002512

10. Chung CY, Wong ES, Liu CCH, Wong MOM, Li KKW. Clinical features and prognostic factors of Klebsiella endophthalmitis-10-year experience in an endemic region. Eye (Lond). 2017;31(11):1569-75. https://doi.org/10.1038/ eye.2017.92.

11. Lange C, Feltgen N, Junker B, Schulze-Bonsel K, Bach M. Resolving the clinical acuity categories "hand motion"and "counting fingers", using the Freiburg Visaul acuity test (FrACT). Graefes Arch Clin Exp Ophthalmol. 2009; 247(1):137-42. https://doi.org/10.1007/s00417-008-0926-0.

12. Yang GY, Huang $X$, Jiang $S, X u Z$. Endogenous endophthalmitis caused by klebsiella pneumonia: a ten-year retrospective study in Western China. Ophthalmic Res, Published online: January 15, 2020.doi: https://doi.org/10. 1159/000505928..

13. Ang M, Jap A, Chee SP. Prognostic factors and outcomes in endogenous Klebsiella pneumoniae endophthalmitis. Am J Ophthalmol. 2011;151(2):33844. https://doi.org/10.1016/j.ajo.2010.08.036.

14. Kashani AH, Eliott D. The emergence of Klebsiella pneumoniae endogenous endophthalmitis in the USA: basic and clinical advances. J Ophthalmic Inflamm Infect. 2013;3(1):28. https://doi.org/10.1186/1869-5760-3-28.

15. Brown JD, Chapman S, Ferquson PE. Blood cultures and bacteraemia in an Australian emergency department: evaluating a predictive rule to guide collection and their clinical impact. Emerg Med Australas. 2017;29(1):56-62. https://doi.org/10.1111/1742-6723.12696.

16. Makusha LP, Young CR, Agarwal DR, Pucar D. Bilateral end-organ Endophthalmitis in setting of Serratia marcescens Urosepsis on 18F-FDG PET/CT. Clin Nucl Med. 2020;45(3):e141-3. https://doi.org/10.1097/RLU. 0000000000002883.

17. Lin YT, Cheng YH, Chuang C, Chou SH, Liu WH, Huang CH, et al. Molecular and clinical characterization of multidrug-resistant and Hypervirulent Klebsiella pneumoniae strains from liver abscess in Taiwan. Antimicrob Agents Chemother. 2020;64(5):e00174-20. https://doi.org/10.1128/AAC. 00174-20

18. Scheer CS, Fuchs C, Grundling M, Vollmer M, Bast J, Bohnert JA, et al. Impact of antibiotic administration on blood culture positivity at the beginning of sepsis: a prospective clinical cohort study. Clin Microbiol Infect. 2019;25(3):326-331. doi.org/https://doi.org/10.1016/j.cmi.2018.05.016.

19. Shields RA, Smith SJ, Pan CK, Do DV. Endogenous klebsiella pneumoniae endophthalmitis in northern California. Retina. 2019;39(3):614-20. https://doi. org/10.1097/IAE.0000000000001994.

20. Damm LJ, Boden KT, Januschowski K. Importance of vitrectomy in endophthalmitis : how immediate vitrectomy can restore visual acuity. Ophthalmologe. 2019;116(6):569-71. https://doi.org/10.1007/s00347-0180768-z.

21. Fine HF, Iranmanesh $R$, Iturralde D, Spaide RF. Outcomes of 77 consecutive cases of 23-gauge transconjunctival vitrectomy surgery for posterior segment disease. Ophthalmology. 2007;114(6):1197-200. https://doi.org/10. 1016/j.ophtha.2007.02.020.

22. Huang $\mathrm{CH}$, Hsieh $\mathrm{YT}$, Yang CM. Vitrectomy for complications of proliferative diabetic retinopathy in young adults: clinical features and surgical outcomes. Graefes Arch Clin Exp Ophthalmol. 2017;255(5):863-71. https:// doi.org/10.1007/s00417-016-3579-4 
23. Ho IV, Guillermo FZ, Levasseur S, Ting E, Liew G, Playfair J, et al. Early pars plana vitrectomy for treatment of acute infective endophthalmitis. Asia Pac J Ophthalmol. 2019;8(1):3-7. https://doi.org/10.22608/APO.2018414.

24. Panchal B, Tyagi M, Pathengay A, Sharma S, Dave VP, Gandhi U, et al. Endophthalmitis following suture removal-clinical outcomes and microbiological profile. Semin Ophthalmol. 2019;34(2):115-23. https://doi. org/10.1080/08820538.2019.1590605.

25. Shen $X, X u$ G. Vitrectomy for endogenous fungal endophthalmitis. Ocul Immunol Inflamm. 2009;17(3):148-52. https://doi.org/10.1080/ 09273940802689396

26. Clarke B, Williamson TH, Gini G, Gupta B. Management of bacterial postoperative endophthalmitis and the role of vitrectomy. Surv Ophthalmol. 2018;63(5):677-93. https://doi.org/10.1016/j.survophthal.2018.02.003.

27. Chen YH, Li YH, Lin YJ, Chen YP, Wang NK, Chao AN, et al. Prognostic factors and visual outcomes of pyogenic liver abscess-related endogenous Klebsiella pneumoniae Endophthalmitis: a 20-year retrospective review. Sci Rep. 2019;9(1):1071. https://doi.org/10.1038/s41598-018-37643-y.

28. Kim SJ, Chu ST, Lee KS, Nam SW, Choi JK, Chung JW, et al. Klebsiella pneumoniae metastatic endophthalmitis and thyroid abscess complicating liver abscess. Clin Mol Hepatol. 2018;24(1):88-91. https://doi.org/10.1093/ ofid/ofu080

29. Chronopoulos A, Schwarz P, Fornoff L, Hattenbach LO. Vitrectomy for endophthalmitis: incubation time and prognosis following intravitreal injection vs. cataract surgery. Ophthalmology. 2019;116(8):746-52. https:// doi.org/10.1007/s00347-018-0816-8.

30. Chen KJ, Chen YP, Chao AN, Wang NK, Wu WC, Lai CC, et al. Prevention of evisceration or enucleation in endogenous bacterial panophthalmitis with no light perception and scleral abscess. PLoS One. 2017;12(1):e0169603. https://doi.org/10.1371/journal.pone.0169603.

31. Chew KL, Lin RTP, Teo JWP. Klebsiella pneumoniae in Singapore: Hypervirulent infections and the Carbapenemase threat. Front Cell Infect Microbiol. 2017;7:515. https://doi.org/10.3389/fcimb.2017.00515.

\section{Publisher's Note}

Springer Nature remains neutral with regard to jurisdictional claims in published maps and institutional affiliations.

Ready to submit your research? Choose BMC and benefit from:

- fast, convenient online submission

- thorough peer review by experienced researchers in your field

- rapid publication on acceptance

- support for research data, including large and complex data types

- gold Open Access which fosters wider collaboration and increased citations

- maximum visibility for your research: over $100 \mathrm{M}$ website views per year

At $\mathrm{BMC}$, research is always in progress.

Learn more biomedcentral.com/submissions 